\title{
Mechanical behavior and infrared imaging of ferromagnetic NiFeGaCo SMA single crystal subjected to subsequent compression cycles
}

\author{
Elzbieta A. Pieczyska
}

Received: 6 September 2013/Accepted: 23 December 2013/Published online: 10 January 2014

(C) The Author(s) 2014. This article is published with open access at Springerlink.com

\begin{abstract}
In this paper, an experimental study of thermomechanical coupling related to pseudoelastic deformation of ferromagnetic shape memory alloy (FSMA) is presented. NiFeGaCo single crystal was subjected to subsequent loading-unloading compression cycles. The stress-strain parameters were recorded by mechanical and laser extensometers. Fast and sensitive infrared camera was used in order to record infrared radiation emitted by the sample surface during the deformation process and to calculate temperature changes related to both exothermic forward and endothermic reverse martensitic transformations. Thanks to the applied techniques, we could investigate a nucleation and development of the stressinduced martensitic transformation. The obtained thermograms exposure localized character of the transformation, initiating in form of inclined bands of higher temperature and developing throughout the sample. The elaborated average temperature change of the SMA sample surface reflects an instantaneous rate of the transformation development. High repeatability of both mechanical and temperature changes obtained in the subsequent loading cycles indicates good thermomechanical properties of the FSMA crystal and confirms high accuracy of the measurement.
\end{abstract}

E. A. Pieczyska (

Institute of Fundamental Technological Research,

Warsaw, Poland

e-mail: epiecz@ippt.pan.pl
Keywords Ferromagnetic shape memory alloy $\cdot \mathrm{NiFeGaCo} \cdot$ Phase transformation . Transformation bands · Mechanical properties . Compression test $\cdot$ Cyclic loading $\cdot$ Infrared camera $\cdot$ Temperature change .

Thermomechanical coupling

\section{Introduction}

The development of shape memory alloy (SMA) applications is currently of great interest due to their ability to join both sensor and actuator functions and that they are almost perfect in shape recovery. Among them, ferromagnetic shape memory alloys, discovered by Ullakko in 1996 [1], exhibit magnetic field- or stress-induced reversible strains of up to $10 \%$. Similarly to classical SMA, these alloys in most cases are characterized by a high-symmetry cubic structure in the high-temperature phase and a low-symmetry tetragonal structure in the low-temperature phase. An actuator using FSMA is rapid and can operate with 50-100 $\mathrm{Hz}$ frequency, whereas the frequency of TiNi SMA is $1 \mathrm{~Hz}$ only [1-4]. Thanks to these advantages, the FSMA are applied as elements of instruments demanding very fast operation. Investigation of mechanical characteristics, the alloys related temperature changes and the manner of the transformation development is crucial for their applications, since the SMA are very sensitive to the temperature changes [58]. Effects of the thermomechanical couplings 
occurring during the stress-induced martensitic transformation under various loadings have recently been studied in several research centers. For e.g., thermomechanical results of NiTi SMA subjected to simple shear are presented in [3], to tension in [4-6] and to compression in [7, 8]. In [6], nucleation and development of the transformation pattern in TiNi SMA was studied by fast infrared camera. In [8], the effects of thermomechanical couplings were studied for ferromagnetic $\mathrm{NiFeGa}$ polycrystalline samples during pseudoelastic compression loading. In [9], the constitutive models were proposed at the continuum level for both the single- and polycrystalline SMA. In [10], the effects of thermomechanical couplings were presented for various kinds of loading, including fatigue and torsion, for shape memory alloys, polymers and composites, yet no results for ferromagnetic shape memory alloys were included.

\section{Experimental procedures}

A ferromagnetic shape memory alloy (NiFeGaCo) single crystal small rectangular samples were subjected to subsequent loading-unloading pseudoelastic compression cycles. The tests were carried out on Instron testing machine, at room temperature, with a strain rate of $10^{-2} \mathrm{~s}^{-1}$ and within $5 \%$ of reversible strain range. During the loading, the mechanical parameters and infrared radiation from the sample surface were simultaneously recorded. The temperature distribution was measured by fast and sensitive Phoenix Flir system IR camera with InSb detector working in the spectral range of 3-5 $\mu \mathrm{m}$. The frame frequency of the image was $538 \mathrm{~Hz}$, the sensitivity of the temperature measurement $0.025 \mathrm{~K}$. The IR system enables obtained infrared photographs, i.e. thermograms, to be stored in digital form. That not only allows reproduction of the images at any time but also makes calculation of the temperature and its presentation straightforward as a function of time, stress, strain or other parameters of the deformation process. In order to assure better thermal isolation, two plates of mica (isinglass) were placed between the sample and the compression grips of the testing machine. Before the test, the sample surface had been covered with a very thin layer of black ink with a high and wellknown coefficient of emissivity in order to obtain higher accuracy of the temperature measurement in

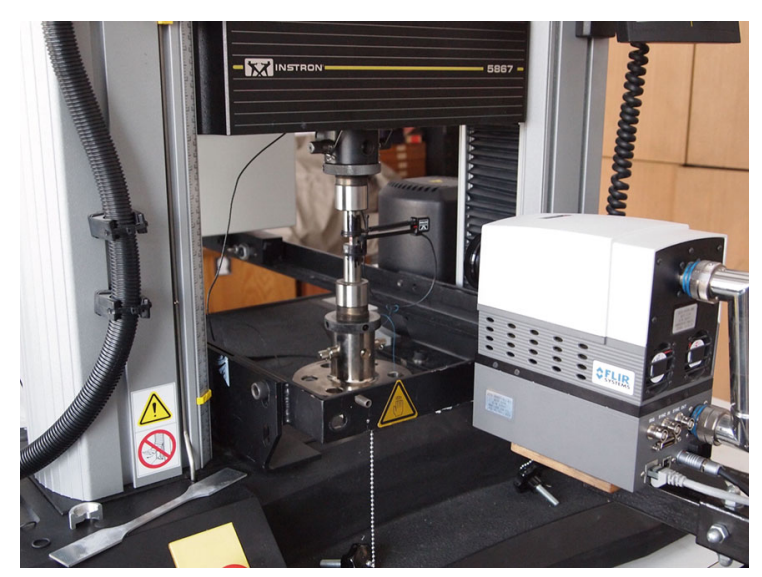

Fig. 1 Photograph of the experimental set up: FSMA single crystal sample in grips of Instron testing machine and FLIR System infrared camera

the initial test. In the initial test the strain parameters were measured by both a mechanical and laser extensometers, acting without influence of the testing machine. However, since the laser beam disturbs the infrared measurement, the laser extensometer was used only during the test for the experimental apparatus validation. The photograph of the experimental set up is shown in Fig. 1.

\section{Material, specimens and loading program}

The FSMA single crystal was cast by arc melting technique. The alloy was characterized by $\mathrm{Ni}_{49} \mathrm{Fe}_{18}$ $\mathrm{Ga}_{27} \mathrm{Co}_{6}$ atomic composition; the structure was FCC and the crystal direction was [100].

Two heating treatments were applied for the FSMA samples before the loading:

- Heating at $1150{ }^{\circ} \mathrm{C}$ during $1 \mathrm{~h}$ and water quenching in order to remove precipitates

- Heating at $800{ }^{\circ} \mathrm{C}$ and slow cooling in order to obtain higher ordering

After that, the alloy was cut into small rectangular pieces of the following sizes:

(1) $3.83 \times 3.95 \times 3.95 \mathrm{~mm}$ (short samples);

(2) $8.99 \times 4.54 \times 3.92 \mathrm{~mm}$ (long samples).

Two experimental loading approaches were applied for the obtained FSMA samples:

(1) Short samples program aimed at the experimental set-up validation 
- 1 st cycle-compression loading with strain rate $10^{-2} \mathrm{~s}^{-1}$ to strain range $5 \%$ and next unloading

- Five subsequent compression loadingunloading cycles with the same strain rate $10^{-2} \mathrm{~s}^{-1}$ and strain $5 \%$

During the loading the stress-strain data were recorded by both the laser and mechanical extensometers.

(2) Long samples program aimed at the estimation of the effects of the thermomechanical couplings:

- 1 st cycle-compression loading with strain rate $10^{-2} \mathrm{~s}^{-1}$ to strain range $5 \%$ and next unloading

- 5 subsequent compression loading-unloading cycles with the strain rate $10^{-2} \mathrm{~s}^{-1}$ and strain $5 \%$.

During the loading the stress-strain data were recorded by the mechanical extensometer and the sample temperature changes were measured by infrared camera.

\section{Results and discussion}

One of the important directions of the research was to check whether the SIMT in NiFeGaCo single crystal occurs macroscopically homogeneously or not. And if the transformation is localized, how it nucleates and develops. As a result, it was found that during the first cycle of the compression loading a band inclined by over $45^{\circ}$ and characterized by higher temperature from the other part of the sample by approximately $0.4 \mathrm{~K}$ appeared manifesting localized character of the transformation (Fig. 2a). At higher strain a second family of bands appears, emerging at the angle close to $90^{\circ}$ according to the loading direction (Fig. 2b), followed by many transformation bands developing towards the upper grip of the testing machine (Fig. 2c). After reaching the grip area, a strong band in the upper part of the sample appeared developing again at the angle close to $45^{\circ}$. The quality of the obtained images is rather low and not to compare to the transformation pattern recorded by the same high quality IR camera and presented in [6]. This is caused by a special ring used here in order to adjust the optical measurement system and small energy received from so small samples. Similar localization effects were recorded in the subsequent loading-unloading cycles. The bands behavior, especially observed on a movie, seams to reflect a compromise between the alloy structure and the sample boundary conditions.

The transformation band shown in Fig. 2d can be noticed with naked eye (Fig. 3a). Nevertheless, the infrared technique, especially film, allows observing and recording also the kinetic effects of the bands origin and evolution. Nucleation and development of the bands are especially valuable in the case of single crystal, since the localization phenomena are not influenced by the grains and by the grain boundaries interactions, so the observed effects are almost direct related to the SIMT mechanisms, according to its
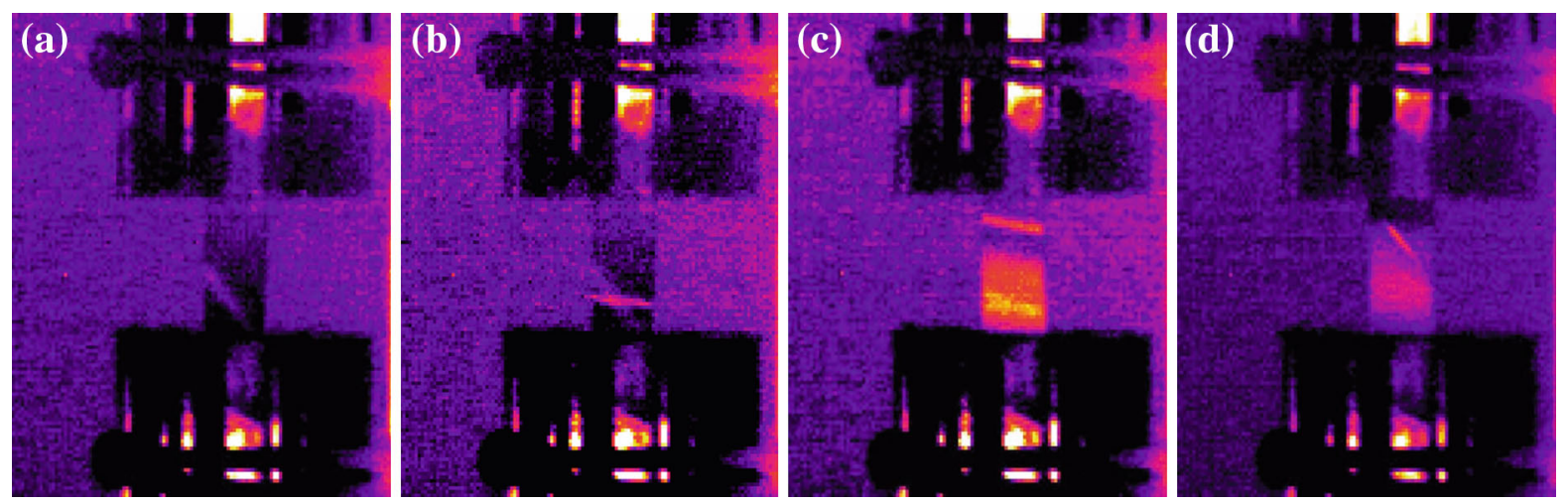

Fig. 2 Subsequent stages of FSMA single crystal compression loading in infrared: a 1st transformation band, b 1st and 2nd bands, c many developing bands, $\mathbf{d}$ a band developing from upper grip 
Fig. 3 a Photograph of FSMA sample in compression: a general view, b infrared thermogram showing a rectangular area on the sample surface used to calculate its average temperature
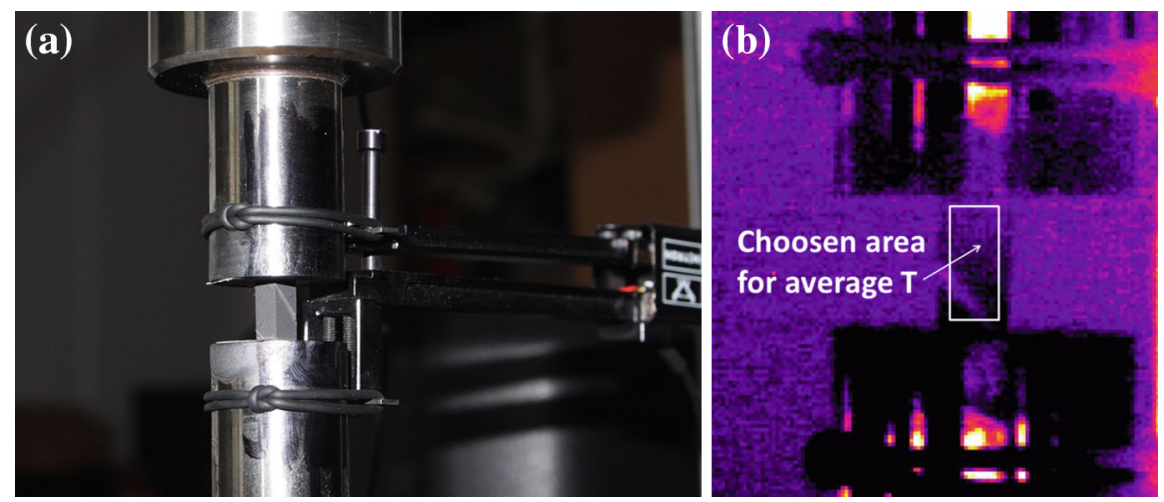

thermodynamics. SMA is very sensitive to the temperature. Therefore, the awareness of the stress and temperature changes accompanied the martensitic transformation is important for their thermodynamics study. In order to estimate the global heat effects, related to the transformation, average values of the sample surface temperature, avoiding the border effects, were calculated (Fig. 3b).

Stress-strain curve and their related average temperature changes elaborated in this manner for NiFeGaCo 1st compression cycle are shown in Fig. 4. The stress and strain quantities were related to the current (instantaneous) values of the sample cross-section, obtaining so-called "true stress" $\sigma_{\text {true }}$ and "true strain" $\varepsilon_{\text {true }}$ values, presented in the diagrams. In addition, the transformation stages observed during the FSMA loading and shown in the thermograms in Fig. 2 were denoted here by a, b, c, d.

Looking at the temperature change versus strain curve shown in Fig. 4 one can note that similarly to what was observed for TiNi SMA [5, 6], the martensitic forward transformation starts before the $\sigma(\varepsilon)$ knee, in this case at $\approx 60 \mathrm{MPa}$, since the temperature starts to increase at this stress level. At the stress $\approx 80 \mathrm{MPa}$ the localized in bands transformation initiates and growths as the stress increases till $100 \mathrm{MPa}$ (Fig. 4). The comparison of the stress-strain curves obtained for the subsequent six compression loading-unloading cycles within strain range $5 \%$ is shown in Fig. 5a. Their related average temperature changes are presented versus strain in Fig. 5b.

The obtained maximal temperature rise is about 4.6 K. The value is quite high, as recorded in the case of so small samples and significant heat flow to the surroundings, first of all to the solid grips of the testing machine. While unloading, the sample temperature

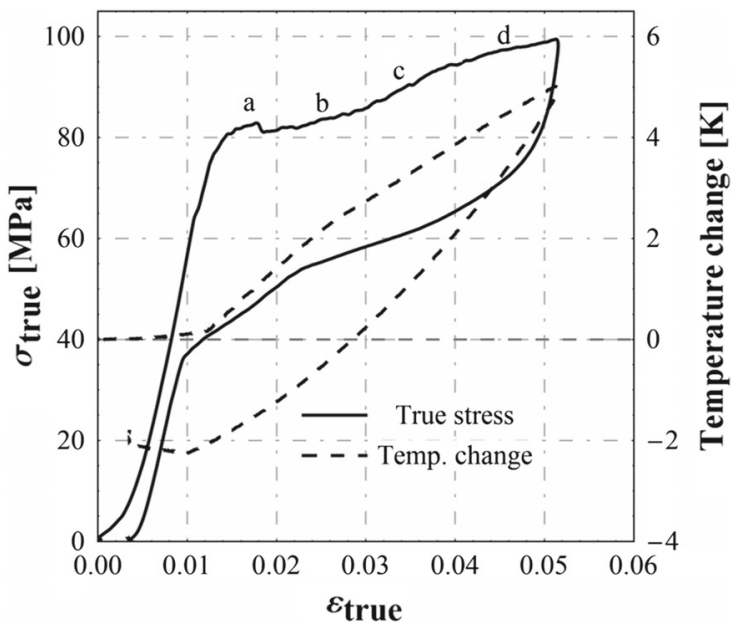

Fig. 4 Stress and temperature change versus strain curve for 1 st compression cycle of FSMA single crystal: $a, b, c, d$ denote transformation stages shown in thermograms presented in Fig. 2

decreases due to the endothermic reverse transformation. Finally, the temperature decreases even below the initial sample temperature by approximately $2.2 \mathrm{~K}$, which is caused mainly by the drop in temperature due to the elastic unloading stage and the endothermic reverse transformation. Looking at Fig. 5a, b it should be noted very high repeatability of the mechanical and temperature results obtained for the subsequent five loading-unloading cycles. The maximal discrepancy noticed between the 1 st and the 2nd cycle, usually observed during the cyclic loading, is mainly caused by the adjusting procedure of the sample, extensometer, grips and testing machine system. The obtained results confirm good quality of the FSMA samples, correctness of the mechanical tests, proper measurement techniques and high sensitivity of the camera. 
(a)
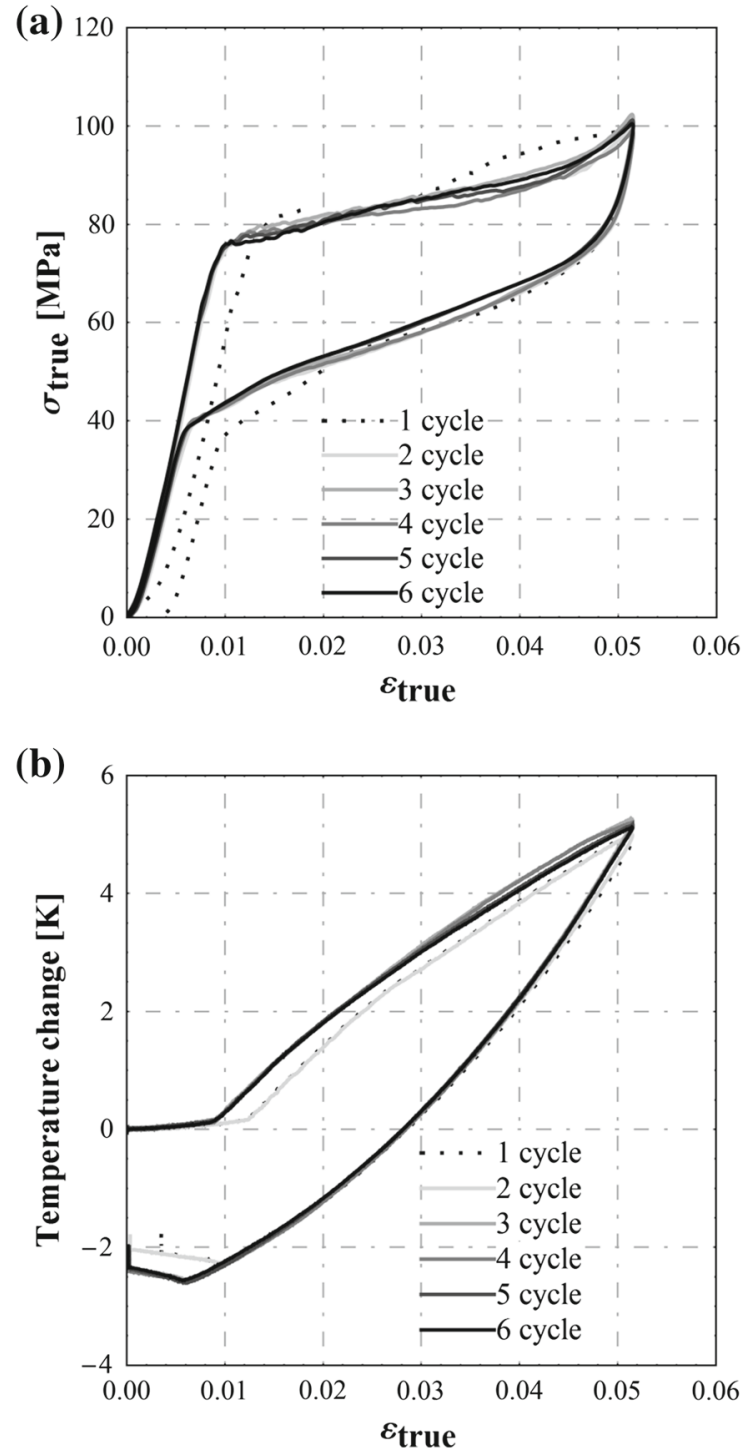

Fig. 5 Stress versus strain (a) and temperature versus strain (b) curves obtained for 6 loading-unloading compression cycles of ferromagnetic $\mathrm{NiFeGaCo} \mathrm{single} \mathrm{crystal}$

\section{Conclusions}

An experimental study of the thermomechanical coupling occurring during pseudoelastic deformation in ferromagnetic $\mathrm{NiFeGaCo}$ single crystal subjected to subsequent compression cycles was presented. Good repeatability of the mechanical and temperature results observed for the cyclic loading confirms high quality of the alloy, correctness of the mechanical tests and high sensitivity of the equipment. It was found that the martensitic exothermic forward transformation nucleates and develops in the inclined transformation bands of higher temperature. The estimated difference of the temperature between the band and rest part of the sample is approximately $0.4 \mathrm{~K}$. The reverse transformation is also not uniform; however the recorded localization effects are not so significant. The localized transformation is also observed in the subsequent loading-unloading compression cycles. The values of the maximal temperature changes related to the exothermic martensitic forward transformation and measured in non-contact manner by high-performance IR camera are about $4.6 \mathrm{~K}$. After the unloading, the sample temperature drop below its initial state by approximately $2.2 \mathrm{~K}$. These thermomechanical coupling effects, observed in SMA and confirmed in FSMA, are important for developing other direction of the alloys applications as cooling elements and will be a subject of our future research.

Acknowledgments The financial support of the Polish National Center of Science under Grant No. 2011/01/M/ST8/ 07754 is acknowledged. Author is grateful to E. Cesari for supplying the FSMA specimens and his valuable advices, to L. Urbanski and R. Maciak for processing mechanical data, to $\mathrm{M}$. Maj for elaborating the temperature changes and to Paulina Pieczyska for linguistic correction of the text.

Open Access This article is distributed under the terms of the Creative Commons Attribution License which permits any use, distribution, and reproduction in any medium, provided the original author(s) and the source are credited.

\section{References}

1. Ullakko K (1996) Magnetically controlled shape memory alloys: a new class of actuator materials. J Mat Eng Perf 5:405-409

2. Santamarta R, Cesari E, Muntasell J, Font J, Pons J, Ochin P (2010) Thermal and microstructural evolution under ageing of several high-temperature $\mathrm{Ni}-\mathrm{Mn}-\mathrm{Ga}$ alloys. Intermetallics 18:977-983

3. Pieczyska EA, Gadaj SP, Nowacki WK, Luckner J, Tobushi $\mathrm{H}$ (2009) Martensite and reverse transformation during simple shear of NiTi shape memory alloy. Strains 45: 93-100

4. Favier D, Louche H, Schlosser P, Orgeas L, Vacher P, Debove L (2007) Homogeneous and heterogeneous deformation mechanisms in austenitic polycrystalline $\mathrm{Ti}-50.8$ at.\% $\mathrm{Ni}$ thin tube in tension. Investigation via temperature and strain fields measurements. Acta Mater 55:5310-5322

5. Pieczyska EA (2010) Activity of stress-induced martensite transformation in shape memory alloy studied by infrared technique. J Mod Opt 57(18):1700-1707 
6. Pieczyska EA, Tobushi H, Kulasiński K (2013) Development of transformation bands in TiNi SMA for various stress and strain rates studied by a fast and sensitive infrared camera. Smart Mater Struct 22(3):035007

7. Pieczyska EA, Tobushi H, Kulasinski K, Takeda K (2012) Impact of strain rate on thermo-mechanical coupling effects in TiNi SMA subjected to compression. Mater Trans 53:1905-1909

8. Pieczyska EA, Dutkiewicz J, Masdeu F, Luckner J, Maciak $\mathrm{R}$ (2011) Investigation of thermomechanical properties of ferromagnetic NiFeGa shape memory alloy subjected to pseudoelastic compression test. Arch Metal Mater 56(2):401-408

9. Wang Jiong, Steinmann Paul, Dai Hui-Hui (2013) Analytical study on the stress-induced phase or variant transformation in slender shape memory alloy samples. Meccanica 48:943-970

10. Tobushi H, Matsui R, Takeda K, Pieczyska E (2013) Mechanical properties of shape memory materials. Materials science and technologies, mechanical engineering theory and applications. NOVA, New York 